\title{
Determination of Velocity and Radius of Supernova Remnant after 1000 yrs of Explosion
}

\author{
Baha T. Chiad1', Lana T. Ali' ${ }^{2}$, Abdhreda S. Hassani ${ }^{1}$ \\ ${ }^{1}$ Department of Physics, College of Science, University of Baghdad, Baghdad, Iraq \\ ${ }^{2}$ Department of Astronomy and Space, College of Science, University of Baghdad, Baghdad, Iraq \\ Email: Lana talib@yahoo.com
}

Received 21 February 2015; accepted 23 June 2015; published 26 June 2015

Copyright (C) 2015 by authors and Scientific Research Publishing Inc.

This work is licensed under the Creative Commons Attribution International License (CC BY). http://creativecommons.org/licenses/by/4.0/

(c) (i) Open Access

\begin{abstract}
Supernova explosions are described as very violent events which transfer a significant amount of energy to interstellar media and are responsible for a large variety of physical processes. This study does not discuss the actual explosion mechanisms but follows the behavior of the dynamical evolution of some selected type I and type II supernova remnant and particularly after a thousand years from their explosion and shows how the density of the medium affects the evolution and the lifetime of each remnant. By studying such behaviors, a simplified model has been proposed here for the velocity and radius of the remnant after thousand years of explosion that depends only on the density of the medium and age of the remnant. It has been found that all types of supernova remnants have similar behaviors after a thousand years from their explosion despite their origin formation. Moreover, it is demonstrated that, when those selected remnants have entered or will enter into their radiative phase, an idea on their physical properties will be obtained.
\end{abstract}

\section{Keywords}

Supernova, Supernova remnant, Radiative Phase

\section{Introduction}

The mass of the star plays a significant role in the determination of its lifetime. Once the star reaches the giant stage, its thermonuclear reaction will speed up and its fate will be determined. A star with mass $<8 \mathrm{M}_{\odot}$ will reach the carbon/oxygen reaction and cease since it has insufficient temperature to ignite further reaction and it ends its life expelling its outer envelope in a planetary nebula and leaving behind a carbon/oxygen white dwarf 
[1]. However, the white dwarf is not always the end product in the collapse of a mid-sized star. If a white dwarf is in a contact binary system it will accrete material from the companion star, and this will continue until its mass exceeds the Chandrasekhar mass limit which is about $1.4 \mathrm{M}_{\odot}$ [2]. At this moment the density and the temperature in the center of the white dwarf become so severe that carbon starts fusing explosively and within one second it will undergo a thermonuclear explosion and is completely destroyed, leaving nothing behind except remnant, producing type Ia supernova [1]. While on the other hand, more massive stars $\left(\geq 8 \mathrm{M}_{\odot}\right)$ will go beyond carbon/oxygen reaction until they reach the iron reaction stage. At this point the thermonuclear reactions are no longer exothermic, and the core cannot support itself against gravity [2]. The core collapses in a few milliseconds, and an energetic shock propagates out through the envelope of the star to produce an energetic display of electromagnetic radiation marking the catastrophic death of this massive star as a core-collapse supernova [3]. At the same time, almost (99\%) of the explosion energy will be released in the form of energetic neutrinos; the remaining energy is converted into kinetic energy, accelerating the stellar material to speed up greater than the speed of sound and causing a shock wave (moving outwards from the central star) which will compress heat and sweep the surrounding ambient gas as it expands [4]. With time, this expanding material from the explosion in addition to the material that blast wave collects as it travels through the interstellar medium, will form a Supernova Remnant (SNR) [5]. According to Green (Green 2009), more than 274 Galactic SNRs (Green 2009) have been recorded, discovered, and detected and well known examples of these are the remnants of Tycho's SN, Kepler's SN, the Cygnus loop, the Crab nebula, and SN1987A that provide delicate visual indication to their violent births [6].

The subject of supernova plays an important role across a wide range of fields in physics and astronomy as they mark the end point in the life of stars and at the same time their remnants mark the formation of the compact objects and the most massive black holes in the universe as well as they play a significant role in enriching the interstellar medium with heavy elements (up to iron). Moreover, they are the source of much of the energy that heats up the interstellar medium and interacting with it [4]. For these reasons, it is essential to study this remarkable subject. In this paper, we investigate the influence of the density of the medium that surrounds some type Ia and type II supernova remnants such as 1006 SNR, G1.9+0.3, Tycho, Kepler, SN1987A, CasA, Crab nebula, Cygnus loop, and IC443 on their evolution. In addition, we provide a model for the velocity and radius of the remnant when they enter the radiative phase (after thousand years of explosion) that depends only on the density of the medium and age of the remnant.

\section{Evolutionary Stages of Supernova Remnant}

Soon after the supernova explosion takes place, its new life as supernova remnant starts. All remnants from the supernovae explosion seem to go through the same evolutionary phases regardless their differences in the original spectra of supernovae when they all expand and evolve through a series of phases [7]. The first phase of those evolutionary phases will begun almost immediately after the explosion when the shock wave from the supernova expands supersonically into the surrounding medium which will accelerate and drive the interstellar material away from the supernova site with a velocity less than the shock wave's initial velocity which is typically in the order of $30,000 \mathrm{~km} / \mathrm{sec}$ [4]. This phase in which the ejecta are expected to be mostly expanding freely known as the free expansion phase which will last for a few hundred years before the remnant enters the second evolutionary stage of its life as supernova remnant which is the adiabatic phase or the Sedov- Tyler phase. At this phase the remnant will sweep up mass equal to its initial mass that ejected from the explosion which made the shock wave to decelerate from $30,000 \mathrm{~km} / \mathrm{sec}$ to about $10,000 \mathrm{~km} / \mathrm{sec}$, while the radius will increase by $R_{s} \propto t^{2 / 5}[8]$.

After about (100 - 1000) years from the beginning of the Sedov-Tyler phase, the temperature of the matter left behind the shock front will be decreased to about $\left(10^{3}-10^{6}\right) \mathrm{K}$, which allows to the recombination process of the nuclei of abundant elements like $\mathrm{C}, \mathrm{N}$ and $\mathrm{O}$ to recombine with electrons. Consequently, the radiation losses go up (via electron transition) [9] and the remnant experiences its forth evolutionary stage which is the radiative expansion phase that begins at $[10]$ :

$$
t=1.9 \times 10^{4} E_{51}^{3 / 14} n^{-4 / 7}(\mathrm{yr})
$$

and with a radius: 


$$
R=16.2 E_{51}^{2 / 7} n^{-3 / 7}(\mathrm{pc})
$$

where $E_{51}$ represents the explosion energy in unit of $10^{51} \mathrm{ergs}$ and $n_{o}$ denotes the hydrogen number density in the surrounding interstellar medium in the unit of 10 atom per $\mathrm{cm}^{3}$.

Once radiative losses become significant, the plasma flow of the remnant will rapidly change over: the gases quickly cool down in the region of the shock front, and it forms a cold dense thin shell behind the radiative shock [9]. This thin dense shell which is formed through the ISM is filled with a low-density, high-pressure interior so it will be driven by the pressure of the hot, roughly isobaric interior in addition to its own momentum. Subsequently, its velocity will be reduced which makes the post shock fluid velocity to approach shock velocity, so the expanding remnant will passes from its blast wave phase to a snowplow phase which is also said to be the pressure-driven snowplow (pds) stage of evolution [11]. The transition from the blast wave phase to the snowplow phase is illustrated in Figure 1 [12].

The remnant will stay in the radiative phase for tens of thousands of years, until the shock has radiated all its energy and the remnant enters the last evolutionary stage of its life as a remnant which is the Dissipative phase. In this phase, the expansion rate slows down; seeding the local neighborhood with heavy elements and the expansion velocity drops to below the local ISM dispersion velocity which is about $10 \mathrm{~km} / \mathrm{sec}$ and becomes indistinguishable from the surrounding medium [13]. In the next section we will obtain model for the expansion velocity and the radius of the remnant for type Ia and type II supernova when it reaches the radiative phase (after about 1000 years from the supernova explosion) which is independent on the supernova explosion energy.

\section{Calculations and Discussion}

Supernova remnants display a variety of shapes and features that are often remarkably influenced by the environment into which the ejected material is expanding. In this paper some remnants of type Ia and type II supernovae such as: 1006 SNR, G1.9+0.3, Tycho, Kepler, IC443, Cygnus loop, Crab nebula, CasA, and SN1987A, the physical properties of which are listed in Table 1, have been taken in order to investigate how the density of the region that surrounds it affects on the expansion rate of the remnant. In order to establish this, the Counting Pixels Method (see ref. [20]) was applied on the images of the remnants that mentioned in Table 1 to determine their current radius which in turn will be substituted in the following equation in order to calculate the explosion energy $(E)$ of each remnant [33]:

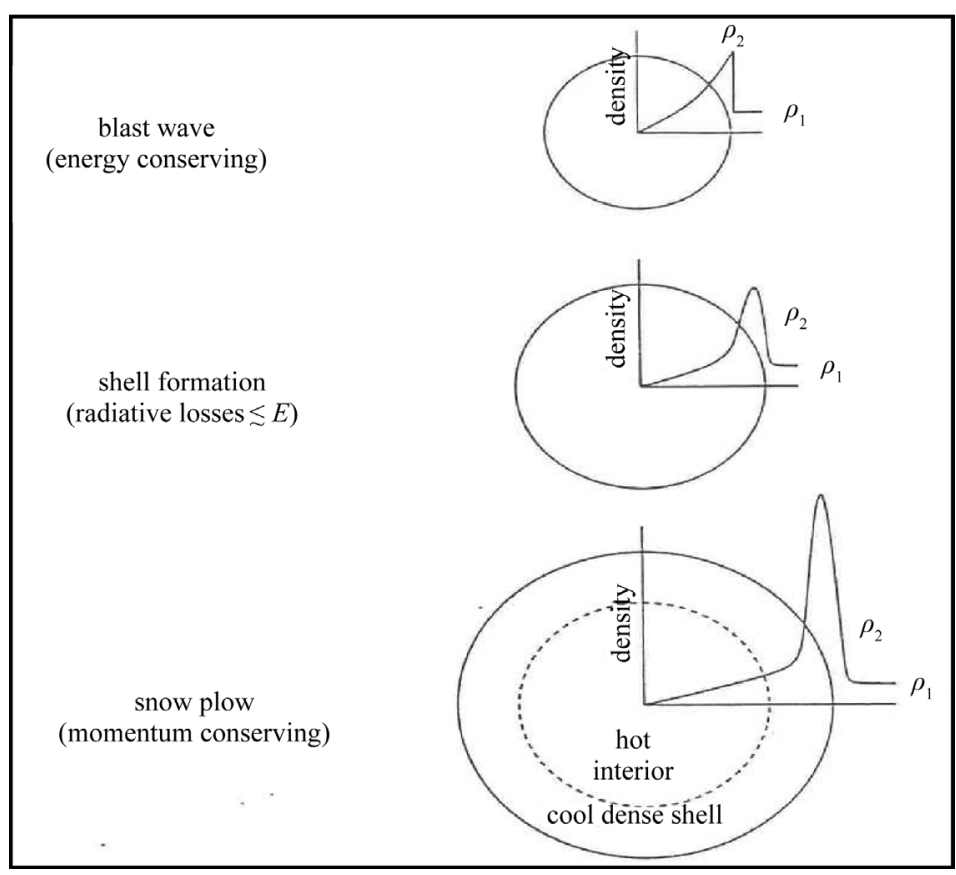

Figure 1. An expanding supernova remnant makes the transition from the blast wave phase (upper image) to the snowplow phase (lower image) [12]. 
Table 1. The physical properties of the selected remnants.

\begin{tabular}{|c|c|c|c|c|c|}
\hline SNR & Age (year) & Distance (pc) & ISM no. density $\left(\mathrm{cm}^{-3}\right)$ & Radius (pc) & Velocity $\left(\mathrm{Km} \cdot \mathrm{s}^{-1}\right)$ \\
\hline G1.9+0.3 & 100 & $8500[14]$ & $0.03[14]$ & $2.2-4.7[14]$ & $14000[14]$ \\
\hline Kepler & 410 & $5000-6400[15]$ & $0.1[16]$ & $2.5-3.8[15]$ & $1550-2000[17]$ \\
\hline Tycho & 442 & $1500-3100[18]$ & $0.2[18]$ & $3.7[18]$ & $1500-2800[18]$ \\
\hline 1006 & 1000 & $2180 \pm 80[19]$ & $0.1[15]$ & $7.1-7.5[19]$ & $2890 \pm 100[19]$ \\
\hline SN 1987A & 26 & $51000[20]$ & $10[21]$ & $0.39[20]$ & $4000[21]$ \\
\hline Cas A & 358 & $3400[22]$ & $1.5[22]$ & $2.6[22]$ & $1000-1500[22]$ \\
\hline Crab & 958 & $2000[23]$ & $0.5[23]$ & $3.4[24]$ & $1400-1500[25]$ \\
\hline Cygnus loop & 17000 & $770[26]$ & $0.1-0.2[27]$ & $21.5-27[28]$ & $200-300[29]$ \\
\hline IC443 & 30000 & $1500[30]$ & $10-20[30]$ & $9.6[31]-15[32]$ & $65-100[30]$ \\
\hline
\end{tabular}

$$
E=3.2 \times 10^{51} n_{o} R^{5} t^{-2}
$$

where $n_{o}$ represents the number density of the surrounding medium, $R$ is the radius of the remnant in unit of $\mathrm{pc}$ and $t$ is the age of the remnant in unit of year.

The output results of this method are listed in Table 2. In terms of the radius, the velocity $(v)$ and the mss $(m)$ of the remnant can also be found by using the following equations:

$$
\begin{aligned}
& v=\frac{R}{t} \\
& m=\frac{4}{3} \pi \rho R^{3}
\end{aligned}
$$

where $\rho$ is the mass density of the interstellar medium.

Moreover, in order to demonstrate the behavior of the radius and the expansion velocity of each remnant with time (which is taken from 1000 years to $30 \times 10^{3}$ years from the explosion) each of Equations (3) and (4) have been applied and the attainable results are plotted in Figure 2(a1) \& Figure 2(a2)) for type Ia SNR and Figure 3(a1) and Figure 3(a2) for type II SNR.

On studying the behavior of Figure 2(a1) and Figure 2(a2)) and Figure 3(a1) and Figure 3(a2) of those selected remnants, we came up with new model of the radius and the expansion velocity of supernova remnant that depend only on the density of the surrounding medium and the age of the remnant which works after thousand year from the explosion, as follow:

$$
\begin{aligned}
& R=0.34332 t^{0.4} n^{-0.2} \\
& V=149666.25 \eta t^{-0.601} n^{-0.2}
\end{aligned}
$$

where $(\eta)$ is the expansion factor which is given in the following equation [34]:

$$
\eta=\frac{v_{b}}{R_{b} / t}
$$

where $v_{b}$, and $R_{b}$ are the velocity and the radius of the blast wave respectively.

Subsequently, the results of applying Equations (6) and (7) have been plotted (Figure 2(a1) and Figure 2(a2) for type Ia SNR and Figure 3(a1) and Figure 3(a2) for type II SNR) and compared with those obtained from Equations (3) and (4) and it was found that, our results are in reasonable agreement with the observed results more than that obtained from applying CPM as shown in Table 3. As well as the results of Table 3 also shows that, our model do not apply to the early evolutionary stages of the SNR as shown in the results of SN1987A and CasA since in these stages the explosion energy is dominant and it accelerates the remnant to higher velocity but with time as the mass of the remnant increases the influence of the explosion energy decreases and the remnant 
Table 2. The result of applying counting pixels method on the selected remnants.

\begin{tabular}{cccccccccc}
\hline & G1.9+0.3 & Kepler & Tycho & $\mathbf{1 0 0 6}$ & SN 1987A & Cas A & $\begin{array}{c}\text { Crab } \\
\text { nebula }\end{array}$ & Cygnus loop & IC443 \\
\hline Radius (pc) & 4.7 & 4 & 5.6 & 18 & 0.49 & 5.055 & 3.41 & 9.6 & 11.1 \\
$\begin{array}{c}\text { Explosion energy } \\
\text { (erg) }\end{array}$ & $0.2 \times 10^{50}$ & $0.02 \times 10^{50}$ & $0.18 \times 10^{50}$ & $6 \times 10^{50}$ & $1.34 \times 10^{51}$ & $0.86 \times 10^{51}$ & $0.015 \times 10^{51}$ & $0.26 \times 10^{51}$ & $0.06 \times 10^{51}$ \\
\hline
\end{tabular}

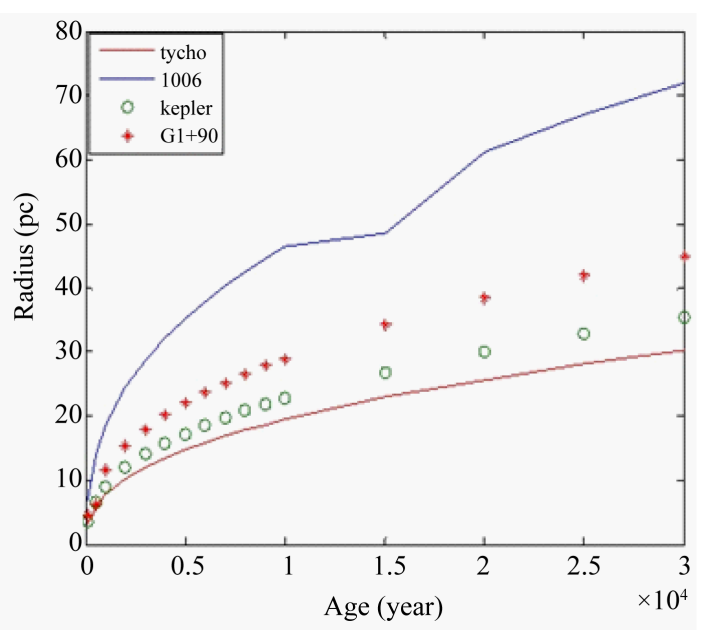

(a1)

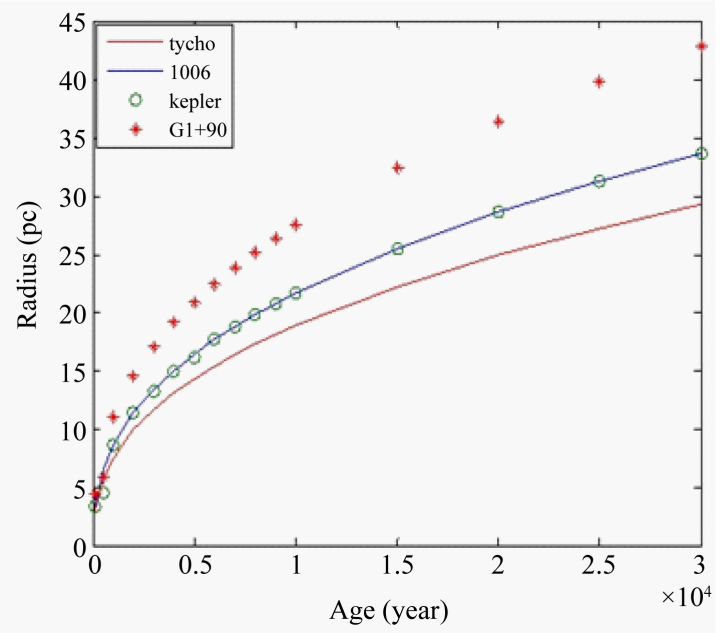

(b1)

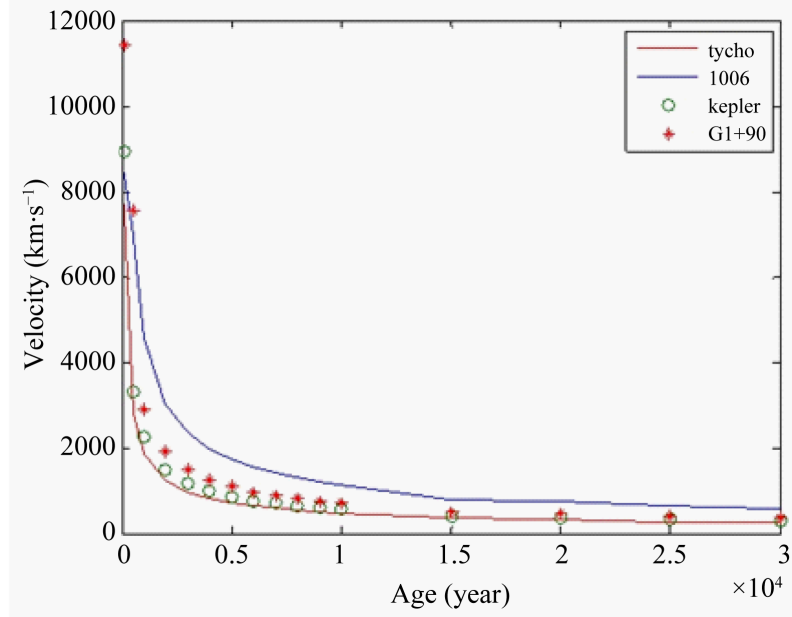

(a2)

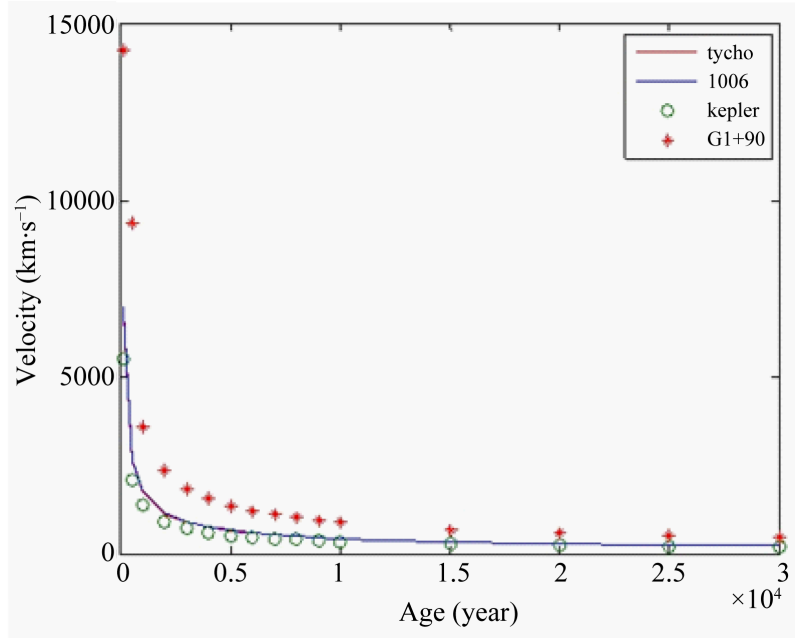

(b2)

Figure 2. Variation of the radius and velocity of Type Ia SNRs with age. (a1) and (a2) by applying Equations (3) and (4), (b1) and (b2) by applying the present model (Equations (6) and (7)).

is decelerated according to one major parameter which is the density of the surrounding interstellar medium as a main parameter affecting the development and determination of the lifetime of each remnants for example the entrance of IC443 to the dens molecular cloud (that lie in the northern portion of the remnant) made the remnant experience an increasing in its mass which may reach a value of $1400 \mathrm{M}_{\odot}$. Consequently, its expansion velocity has been reduced to a value of $83 \mathrm{Km} \cdot \mathrm{s}^{-1}$ and with current radius $13.4 \mathrm{pc}$. In addition to that, each of Equations (6) and (1) can be used in order to find when each remnants of type II supernova enters to radiative phase and what its physical properties will be. When those equations were applied on our selected remnants, we came up with the results that listed in Table 4 which show that (for example) the remnants of IC 443 undergoes into a diative phase sooner than the other remnants even sooner than SN1987A even though they have the same inraterstel- 


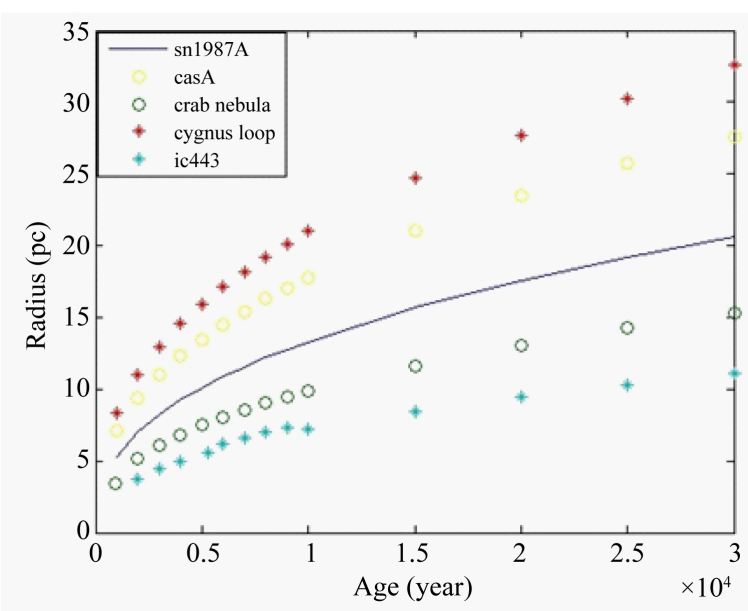

(a1)

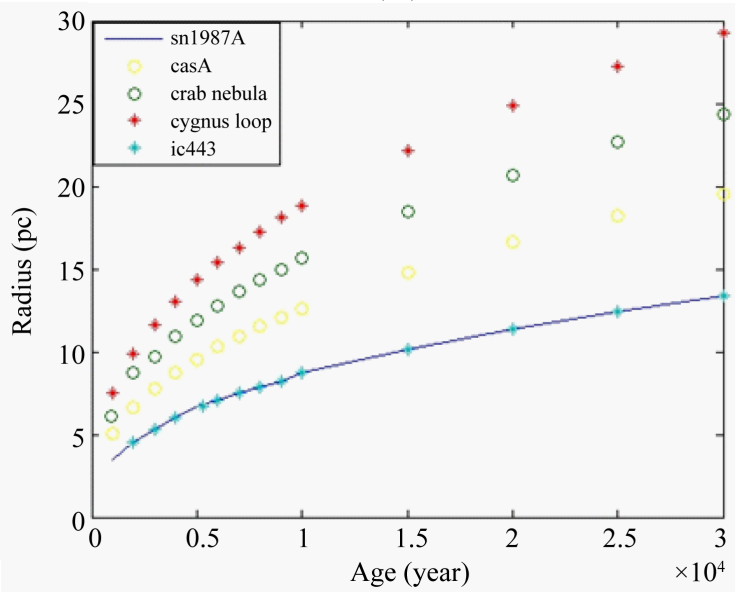

(b1)

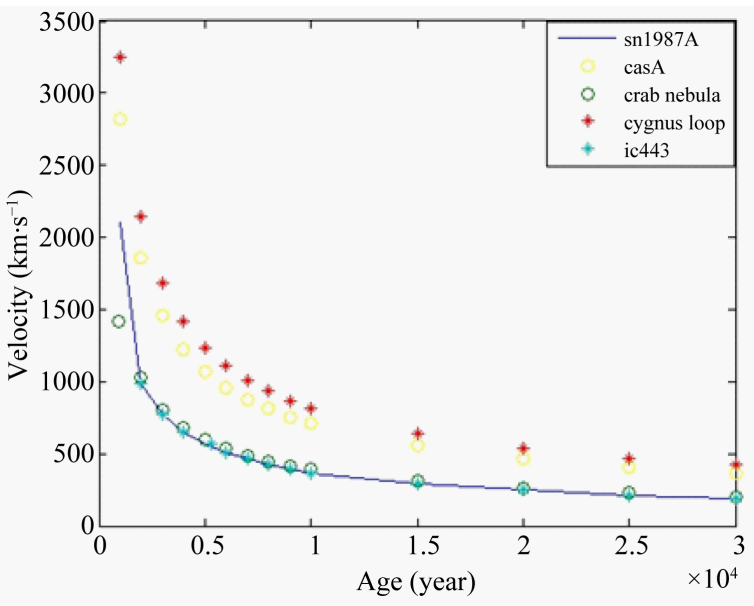

(a2)

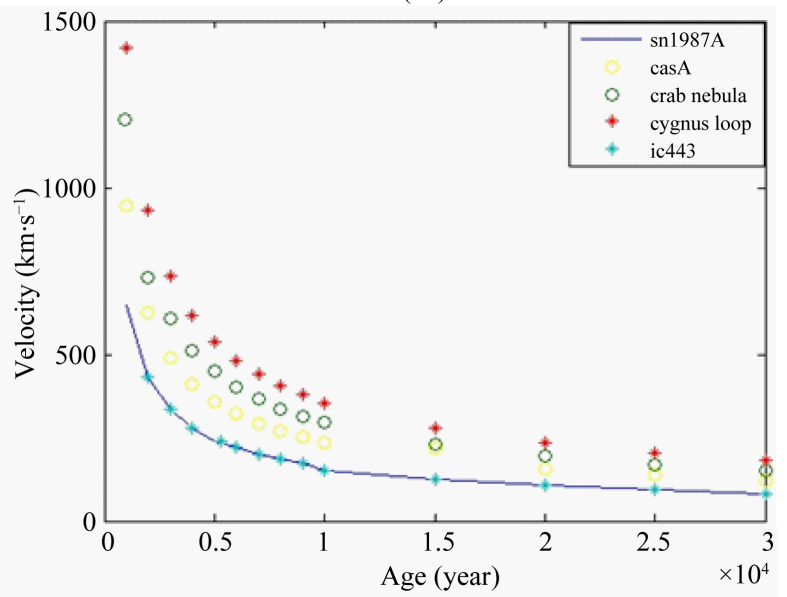

(b2)

Figure 3. Variation of the radius and velocity of Type II SNRs with age. (a1) and (a2) by applying Equations (3) and (4) while (b1) and (b2) by applying the present model (Equations (6) and (7)).

Table 3. Comparison between the present CPM. and our model results and others published elsewhere.

\begin{tabular}{ccccccc}
\hline & \multicolumn{3}{c}{ Velocity $\left(\mathbf{K m}^{-1} \mathbf{~}^{-1}\right)$} & \multicolumn{2}{c}{ Radius (pc) } \\
\cline { 2 - 6 } SNR & Our & CPM. & Researchers & Our & CPM. & Researchers \\
G1.9+0 & 14200 & 11700 & $14000[14]$ & 4.37 & 4.7 & $4.7[14]$ \\
kepler & 2360 & 2420 & $1550-2000[17]$ & 6 & 4 & $4[15]$ \\
Tycho & 2442 & 3147 & $1500-2800[18]$ & 5.4 & 5.6 & $3.7[18]$ \\
1006 & 1755 & 4436 & $2890 \pm 100[19]$ & 8.6 & 18 & $7.1-7.5[19]$ \\
SN 1987A & 6246 & 4113 & $4000[21]$ & 0.78 & 0.49 & $0.39[20]$ \\
Cas A & 1755 & 3872 & $1000-1500[22]$ & 3.32 & 5.055 & $2.637[22]$ \\
Crab & 1220 & 1414 & $1500[25]$ & 6.1 & 3.41 & $3.4[24]$ \\
Cygnus loop & 260 & 224 & $200-300[29]$ & 23 & 9.6 & $20-25[28]$ \\
IC443 & 83 & 101 & $65-100[30]$ & 13.4 & 11.1 & $9.6[31]-15[32]$ \\
\hline
\end{tabular}


Table 4. The physical properties of the selected remnants at the beginning of the radiative phase.

\begin{tabular}{ccccccccccc}
\hline Parameter & Unit & G1.9+0 & kepler & Tycho & $\mathbf{1 0 0 6}$ & SN 1987A & Cas A & $\begin{array}{c}\text { Crab } \\
\text { nebula }\end{array}$ & $\begin{array}{c}\text { Cygnus } \\
\text { loop }\end{array}$ & IC443 \\
\hline $\mathbf{t}_{\text {beginning of radiative phase }}$ & $\mathrm{Yr}$ & $22.7 \times 10^{4}$ & $7 \times 10^{4}$ & $7.5 \times 10^{4}$ & $24 \times 10^{4}$ & $2 \times 10^{4}$ & $1.46 \times 10^{4}$ & $4.28 \times 10^{4}$ & $3.57 \times 10^{4}$ & $1.04 \times 10^{4}$ \\
$\mathbf{R}$ & $\mathrm{Pc}$ & 63.9 & 19.7 & 27.5 & 100.7 & 17.6 & 13 & 17.62 & 22 & 7.25 \\
$\mathbf{V}$ & $\mathbf{K m} \cdot \mathbf{s}^{-1}$ & 280 & 279.5 & 364 & 417 & 394 & 355 & 163.6 & 244.6 & 277 \\
$\mathbf{T}$ & $\mathrm{Kelvin}$ & $1.8 \times 10^{6}$ & $1.77 \times 10^{6}$ & $3 \times 10^{6}$ & $3.9 \times 10^{6}$ & $3.5 \times 10^{6}$ & $2.86 \times 10^{6}$ & $0.6 \times 10^{6}$ & $1.35 \times 10^{6}$ & $1.7 \times 10^{6}$ \\
$\mathbf{M}$ & $\mathrm{M}_{\odot}$ & 255 & 25 & 135 & 3321 & 557 & 336 & 279.5 & 217.6 & 389 \\
\hline
\end{tabular}

lar density. The reason behind that, is the relatively lower explosion energy of IC 443 made it entered to the radiative phase earlier than the remnant of SN1987A. Which means that, not only the density of the medium determine the transition time to the radiative phase but there is another is another important parameter that play a significant role in this transition which is the explosion energy as demonstrated in the result of the remnant of Crab nebula and Cygnus loop which they will take longer time in order to enter into radiative phase according to their explosion energy and interstellar density.

\section{Conclusion}

The aim of this study is to show that all the remnants of supernovae have the same behavior as each other after 1000 years from the explosion but are shifted up or down depending on the one and the only parameter that effects the development and determination of the lifetime of each remnant which is the interstellar density in which they explode since after this time the explosion energy will not have the strength influence on the expansion and specially in the radiative phase. According to our model, we can calculate the expansion velocity and the radius of any supernova remnant (type Ia and type II) after 1000 years from its explosion by knowing only the density of the medium. When we applied our model on those selected remnant, we indicated that SNR which exploded under low density environment would expand freely and take almost a uniform shape (as almost all type Ia SNR and some of type II SNR such as Crab nebula, CasA). And it takes longer time in order to enter the radiative phase. On the other hand, the remnant that evolves primarily in the interclump medium, which has a density $\mathrm{n}=(5-25) \mathrm{H}$ atoms per $\mathrm{cm}^{3}$ such as IC443 (one of the best studied cases of supernova remnants interacting with surrounding molecular clouds) and SN1987A (that surrounded by a region with presupernova mass loss), will have ununiform shape. Since it interacts with the dense clumps, the molecular shock fronts is driven by a considerable overpressure (pressure driven snowplow) compared with the pressure in the rest of the remnant, and thus the remnant enters the radiative phase earlier than other remnants

\section{References}

[1] Katunari'c, J. (2009) Massive Stars: Life and Death. Dissertation, The Ohio State University, Columbus.

[2] Evans, J. (1998) Death of Stars. Vol. 103, Physics \& Astronomy Department, George Mason University, Fairfax.

[3] Arny, T. (1998) Exploration: An Introduction to Astronomy. 2nd Edition, McGraw-Hill Companies, New York, 390391.

[4] NASA'S HEASARC: Education and Public Information (2011) Introduction to Supernova Remnants.

[5] Roger, R. and Landecker, T. (1988) Supernova Remnants and the Interstellar Medium. Cambridge University Press, Cambridge, 12-20.

[6] Lee, J-J., Koo, B-C., Snell, R., et al. (2012) Identification of Ambient Molecular Cloud Associated with Galactic Supernova Remnant IC443.

[7] Theiling, M. (2009) Observation of Very High Energy Gamma Ray Emission from Supernova Remnants with VERITAS. Ph.D. Thesis, Clemson University, Clemson.

[8] Andrew, W. (2006) Filamentary H $\alpha$; Structure in the Milky Way. Ph.D. Thesis, University of Wollongong, Wollongong.

[9] Hnatyk, B., Petruk, O. and Telezhyns'kyi, I. (2007) Transition of Supernova Remnant from the Adiabatic Stage of Evolution to Radiative Stage. Analytical Description. Kinematics and Physics of Celestial Bodies, 23, 137-146 
[10] Candel, I. (2012) Search for Gamma-Ray Emission from Supernova Remnants with the Fermi/LAT and MAGIC Telescopes. Ph.D. Thesis, University of Aut onoma, Barcelona.

[11] Cioffi, D., McKee, C. and Bertschinger, E. (1988) Dynamics of Radiative Supernova Remnants. Astrophysical Journal, 334, 252-265. http://dx.doi.org/10.1086/166834

[12] Shu, F. (1992) The Physics of Astrophysics Volume II: Gas Dynamics. University Science Books, Mill Valley.

[13] Ali, L. (2011) Theoretical Study of the Physical Parameters of the Supernova 1987A. Master's Thesis, University of Baghdad, Baghdad.

[14] Ksenofontov, L.T., Voelk, H.J. and Berezhko, E.G. (2010) Non Thermal Properties of Supernova Remnant G1.9+0.3. http://arxiv.org/abs/1004.2555

[15] Patnaude, D.J., Badenes, C., Park, S. and Laming, J.M. (2012) The Origin of Kepler's Supernova Remnant. http://arxiv.org/abs/1206.6799

[16] Tang, Z.M. (1986) The Dynamic Evolution of the Kepler Supernova Remnant. Astrophysics and Space, 124, 315-327. http://dx.doi.org/10.1007/BF00656043

[17] Blair, W.P., Long, K.S. and Vancura, O. (1991) A Detailed Optical Study of Kepler's Supernova Remnant. Astrophysical Journal, 366, 484-494. http://dx.doi.org/10.1086/169583

[18] Hughes, J.P. (2000) The Expansion of the X-Ray Remnant of Tycho's Supernova (SN 1572). The Astrophysical Journal, 545, L53-L56. http://dx.doi.org/10.1086/317337

[19] Winkler, P.F., Gupta, G. and Long, K.S. (2003) The SN 1006 Remnant: Optical Proper Motions, Deep Imaging, Distance, and Brightness at Maximum. The Astrophysical Journal, 585, 324-335. http://dx.doi.org/10.1086/345985

[20] Chiad, B.T., Karim, L.M. and Ali, L.T. (2012) Study the Radial Expansion of SN 1987A Using Counting Pixels Method. International Journal of Astronomy and Astrophysics, 2, 199-203. http://dx.doi.org/10.4236/ijaa.2012.24025

[21] Dwarkadas, V.V. (2006) Supernova Explosions in Winds and Bubbles with Applications to SN 1987A. http://arxiv.org/abs/astro-ph/0612665

[22] Hwang, U. and Laming, J.M. (2011) A Chandra X-Ray Survey of Ejecta in the Cassiopeia A Supernova Remnant. http://arxiv.org/abs/1111.7316

[23] Henry, R.C., Fritz, G., Meekins, J.F., Chubb, T.A. and Friedman, H. (1972) Absorption of Crab Nebula X-Ray. Astrophysical Journal, 174, 389-397. http://dx.doi.org/10.1086/151498

[24] http://en.wikipedia.org/wiki/Crab Nebula

[25] Bietenholz, M.F., Kronberg, P.P., Hogg, D.E. and Wilson, A.S. (1991) The Expansion of the Crab Nebula. Astrophysical Journal, 373, L59-L62. http://dx.doi.org/10.1086/186051

[26] Blair, W.P., Sankrit, R., Raymond, J.C. and Long, K.S. (1999) Distance to the Cygnus Loop from Hubble Space Telescope Imaging of the Primary Shock Front. The Astronomical Journal, 118, 942-947. http://dx.doi.org/10.1086/300994

[27] Martinez, A.P. (2010) The Cygnus Loop: A Weak Core-Collapse SN in Our Galaxy. Astronomy \& Astrophysics, 527, A55. http://dx.doi.org/10.1051/0004-6361/201015213

[28] Nemes, N. (2005) XMM-Newton Observation of the Northeastern Limb of the Cygnus Loop Supernova Remnant. Ph.D. Thesis, Osaka University, Osaka.

[29] Kirshner, R.P. and Taylor, K. (1976) High-Velocity Gas in the Cygnus Loop. The Astronomical Journal, 208, L83-L86. http://dx.doi.org/10.1086/182237

[30] Rho, J., Jarrett, T.H., Cutri, R.M. and Reach, W.T. (2001) Near-Infrared Imaging and [O I] Spectroscopy of IC 443 Using 2MASS and ISO. The Astrophysical Journal, 547, 885-898. http://dx.doi.org/10.1086/318398 http://arxiv.org/abs/astro-ph/0010551

[31] Hnatyk, B. and Petruk, O. (1998) Supernova Remnants as Cosmic Ray Accelerators. SNR IC 443. Condensed Matter Physics, 1, 655-667. http://dx.doi.org/10.5488/CMP.1.3.655

[32] Zhang, Z.Y., Gao, Y. and Wang, J.Z. (2010) CO Observation of SNR IC 443. Science China: Physics, Mechanics \& Astronomy, 53, 1357-1369.

[33] Lee, J.J., Koo, B.C., Yun, M.S., Stanimirović, S., Heiles, C. and Heyer, M. (2008) A 21 cm Spectral and Continuum Study of IC443 Using the Very Large Array and the ARECIBO Telescope. The Astronomical Journal, 135, 796-808. http://dx.doi.org/10.1088/0004-6256/135/3/796

[34] Truelove, J.K. and Mckee, C.F. (1999) Evolution of Nonradiative Supernova Remnants. The Astrophysical Journal Supplement Series, 120, 299-326. http://dx.doi.org/10.1086/313176 